\title{
The relevance of clinical and radiographic features of jaw lesions: A prospective study
}

\section{Juliane Piragine ARAUJO(a) Celso Augusto LEMOS(a) Thais Gimenez MINIELLO(a) Fabio Abreu ALVES(b)}

(a) Universidade de São Paulo - USP, School of Dentistry, Stomatology Department, São Paulo, SP, Brazil.

(b)AC Camargo Center, Stomatology Department, São Paulo, SP, Brazil.

Declaration of Interests: The authors certify that they have no commercial or associative interest that represents a conflict of interest in connection with the manuscript.

Corresponding Author:

Fábio Abreu Alves

E-mail: falves@accamargo.org.br

DOI: 10.1590/1807-3107BOR-2016.vol30.0096

Submitted: November 30, 2015

Accepted for publication: May 23, 2016

Last revision: June 08, 2016
Abstract: The study was carried out in a Brazilian population and the aim was to describe the prevalence and the clinic-radiographical features of jaw lesions. In addition, a comparison between the main diagnosis hypothesis and final diagnosis was accessed. A prospective study which evaluated all patients with jaw lesions diagnosed in an Oral Diagnosis Center, between August 2013 and October 2014. A total of 450 patients were observed for the first time, and 130 had some type of jaw lesion. The mean age of the patients was 35.2 years \pm 17.86 . Among these, 71 were women (54.62\%) and 87 were Caucasian (66.92\%). The mandible was affected more frequently $(71.43 \%)$ than the maxilla (28.57\%). Swelling and pain were the most frequent clinical signs and symptoms and were observed in $60(42.85 \%)$ and $38(27.14 \%)$ cases, respectively. The panoramic $x$-ray was the main radiographic exam utilized (88.57\%). Radiolucent lesions accounted for 89 cases $(63.57 \%)$ and the unilocular form was present in 114 cases (81.43\%). A total of 93 cases had histopathological analyses and the periapical cyst was the most frequent lesion. In the other 47 lesions, the diagnosis was conducted by clinical and radiographic management. Bone lesions were frequent, being noted on first visit in approximately $30 \%$ of patients; in $1 / 3$ of the cases, the diagnoses were completed with a combination of clinical and radiographic exams.

Keywords: Jaw; Odontogenic Tumors; Odontogenic Cysts; Biopsy; Bone Diseases.

\section{Introduction}

Studies of jaw lesions (JL) have a fundamental role in dentistry where the dentist is, in most cases, responsible for its diagnosis and treatment. Interestingly, most of these lesions are usually discovered during routine radiographs or when the patient reports a swelling in their jaw and a panoramic $x$-ray is performed. In general, painful symptoms are not a common finding. ${ }^{1}$

Most of the information regarding JL is from retrospective studies, which evaluated histopathological lab data. The prevalence of odontogenic tumors, mainly ameloblastomas and keratocystic odontogenic tumors (KOT), has been studied in many parts of the world, and differences in prevalence are observed between countries and between center types (Medical and Dental). African studies show a higher prevalence of ameloblastoma than North 
American studies. ${ }^{2,3,4}$ Fregnani et al. ${ }^{5}$ (2002) concluded that ameloblastomas are the main odontogenic tumor treated in medical hospitals, whereas odontomas are observed more frequently in dentistry clinics and dental hospitals. However, for a large number of other jaw lesions, such as osseous dysplasia (OD), bone sclerosis, and simple bone cysts, histopathological exams are not typically performed, and their prevalence is not well established. Consequently, a prospective analysis (determination of prevalence is more reliable) with descriptiveepidemiology and clinical and radiographic correlation is essential to determine the actual frequency of JL.

The aim of the present study was to evaluate the clinical and radiographic features of JL diagnosed in a single Institution. In addition, the rate of correct diagnosis was also assessed through comparison of the main diagnosis hypothesis (performed by a stomatologists) with the final diagnosis of each case.

\section{Methodology}

This prospective observational study took place at the Oral Diagnosis Center. All of the consecutive patients who had jaw lesions were recruited between August 2013 and October 2014. The Ethics Committee of the Institution approved this research (no. 350.887). All of the patients signed the informed consent and received a copy.

For diagnosis or for biopsy planning, one or multiple radiographic exams were performed according to the lesion indication. Indeed, some data were collected from $x$-ray (periapical, occlusal and panoramic) or helical computed tomography (CT).

\section{Research criteria}

The inclusion criteria included patients with JL observed during the period of the study and came to our clinic for the first time.

The following clinical and radiographic features were collected:

a. clinical: demographic data, signs (mucosal ulceration, bleeding, purulent drainage, and swelling) and symptoms (pain and paresthesia);

b. imaging: site of the lesion, radiodensity/ radiolucency/mixed, unilocular/multilocular form, relationship of the lesion with dental apex, root resorption, dental displacement, impacted tooth, displacement of the mandibular canal, and expansion and/or disruption of the cortical bone. The maxilla and mandible were divided to describe the lesion site:

a. the maxilla was divided into two anatomic regions: anterior (right canine to the left canine) and posterior (first premolar to the maxillary tuberosity);

b. the mandible was divided into five anatomic regions: symphysis (right canine to the left canine), body, angle, ramus, condylar and coronoid processes.

In cases for which biopsy was indicated, the specimens were submitted to the Oral Pathology Lab of the same Institution. The odontogenic cysts and tumors were classified according to the World Health Organization (WHO) criteria. ${ }^{6,7}$ To evaluate the rate of correct diagnosis, the main diagnosis hypothesis was compared to the final diagnosis (histopathological exam). The hypothesis was elaborated by a graduate student and a professor (stomatologists) simultaneously. The cases that did not receive a surgical procedure were excluded and divided into 2 subgroups:

a. Group 1: lesions with histopathological confirmation;

b. Group 2: lesions without histopathological evaluation but for which a surgical procedure was performed and no material (tissue) were collected.

\section{Statistical analysis}

Descriptive statistics were used to report the results. Qualitative variables were presented in absolute and relative frequencies. The main summary measures of the mean, standard deviation, minimum and maximum values for quantitative variables were presented. The statistical software $R$ version 3.0.1 was used for analyses.

\section{Results}

\section{Clinical features}

A total of 997 patients were evaluated during the study period ( 15 months). A total of 450 patients (approximately 45\%) were observed for the first time, and $130(29 \%)$ of the latter had a JL. 
A total of $140 \mathrm{JL}$ were observed in the 130 patients. Of the patients, 71 were women (54.62\%), and 59 were men (45.38\%). The patient ages ranged between 8 and 77 years (mean $35.2 \pm 17.86$ ), and most patients were Caucasian (66.92\%). Regarding the location of the lesions, 3 florid osseous dysplasias and 2 bone scleroses affected both the mandible and the maxilla. Of the other 135 lesions, 95 (67.86\%) were located in the mandible and 40 were in the maxilla (28.57\%). It is noteworthy that some extensive lesions reached more than one region. In the maxilla, 6 cases involved the anterior and posterior region; in the mandible, 8 lesions involved the symphysis and body, 6 involved the body, angle and ramus, and 1 lesion affected the condylar and coronoid processes. The most frequently signs and symptoms were swelling in 60 cases $(42.85 \%)$, pain in 38 cases $(27.14 \%)$, and purulent drainage in 16 cases (11.43\%). Other less frequent included bleeding and ulceration of the mucosa adjacent to the bone (Table 1 ).

\section{Radiographic features}

The panoramic $x$-ray was the exam most frequently used and was performed in 124 cases, followed by periapical x-ray (55 cases), CT (29 cases) and occlusal $\mathrm{x}$-ray (27 cases). Considering the radiographic aspect, 89 out of 140 lesions $(63.57 \%)$ were exclusively radiolucent, $34(24.29 \%)$ were exclusively radiopaque, and 17 cases (12.14\%) were computed as mixed lesions (Figure 1).

A total of 106 lesions (only mixed and radiolucent lesions) were considered for the analysis of the unilocular or multilocular pattern. Of these, 95 were unilocular (84 radiolucent and 11 mixed) and 11 were multilocular ( 5 radiolucent and 6 mixed). The tooth association showed that 101 lesions (72.14\%) had involvement of the dental apex, and 4 of these caused root resorption (1 calcifying cystic odontogenic tumor - CCOT, 1 mucoepidermoid carcinoma - MEC, 1 dentigerous cyst and 1 ameloblastoma), and 23 cases (16.43\%) were associated with an impacted tooth (12 dentigerous cysts, 4 odontomas, 4 KOT, 2 CCOT and 1 ameloblastoma). Dental displacement was observed in 20 lesions (10 periapical cysts, 3 odontomas, 2 KOT, 2 ameloblastomas, 2 dentigerous cysts and 1 CCOT).

The following data were evaluated only for the 29 CT: cortical bone expansion was observed in 11 cases (37.93\%, 3 periapical cysts, 2 dentigerous cysts, 2 KOT,
Table 1. General characteristics of 130 patients who had 140 jaw bone lesions.

\begin{tabular}{|c|c|c|}
\hline Variables & $\mathrm{N}$ & (\%) \\
\hline \multicolumn{3}{|l|}{ Gender $^{a}$} \\
\hline $\mathrm{F}$ & 71 & 54.62 \\
\hline$M$ & 59 & 45.38 \\
\hline \multicolumn{3}{|l|}{ Age (years) ${ }^{a}$} \\
\hline Minimum & 8 & - \\
\hline Maximum & 77 & - \\
\hline Mean & $35.22 \pm 17.86$ & - \\
\hline \multicolumn{3}{|l|}{ Race $^{a}$} \\
\hline Caucasian & 87 & 66.92 \\
\hline Afro descendant & 43 & 33.08 \\
\hline \multicolumn{3}{|l|}{ Maxilla lesions ${ }^{b}$} \\
\hline Anterior & 26 & 57.78 \\
\hline Anterior and posterior & 6 & 33.33 \\
\hline Posterior & 13 & 28.89 \\
\hline \multicolumn{3}{|l|}{ Mandible lesions } \\
\hline Symphysis & 14 & 14.00 \\
\hline Body & 71 & 71.00 \\
\hline Symphysis and body & 8 & 8.00 \\
\hline Body/angle and ramus & 6 & 6.00 \\
\hline Condylar and coronoid processes & 1 & 1.00 \\
\hline \multicolumn{3}{|l|}{ Number of lesions ${ }^{b}$} \\
\hline 1 lesion & 121 & 93.08 \\
\hline 2 lesions & 8 & 6.15 \\
\hline 3 lesions & 1 & 0.77 \\
\hline \multicolumn{3}{|l|}{ Signs and Symptoms } \\
\hline \multicolumn{3}{|l|}{ Swelling ${ }^{b}$} \\
\hline No & 80 & 57.15 \\
\hline Yes & 60 & 42.85 \\
\hline \multicolumn{3}{|l|}{ Pain $^{b}$} \\
\hline No & 102 & 72.86 \\
\hline Yes & 38 & 27.14 \\
\hline \multicolumn{3}{|l|}{ Oral ulcer } \\
\hline No & 138 & 98.57 \\
\hline Yes & 2 & 1.43 \\
\hline \multicolumn{3}{|l|}{ Oral bleeding ${ }^{b}$} \\
\hline No & 134 & 95.71 \\
\hline Yes & 6 & 4.29 \\
\hline \multicolumn{3}{|l|}{ Pus discharge ${ }^{b}$} \\
\hline No & 124 & 88.57 \\
\hline Yes & 16 & 11.43 \\
\hline \multicolumn{3}{|l|}{ Paresthesia ${ }^{b}$} \\
\hline No & 138 & 98.57 \\
\hline Yes & 2 & 1.43 \\
\hline
\end{tabular}

a: Data of 130 patients; $^{\text {b: }}$ Data of 140 bone jaw lesions. 
1 odontoma, 1 bone exostosis, 1 residual cyst, and 1 ossifying fibroma), cortical disruption in 6 cases (20.69\%, 4 periapical cysts, 1 KOT and 1 osteonecrosis) and displacement of the mandibular canal in 3 cases (10.34\%, 1 dentigerous cyst, 1 KOT and 1 osteomyelitis).

\section{Surgical data and diagnosis}

Surgical procedures were performed for 105 lesions and included 20 incisional biopsies, 73 lesion enucleations and 12 surgical interventions for bleeding stimulation (all cases were simple bone cysts). It is noteworthy that in the latter cases, there was no material available for histopathological analysis. In the other 35 lesions, the diagnosis was performed according to the clinical and imaging features, and biopsy was not indicated.
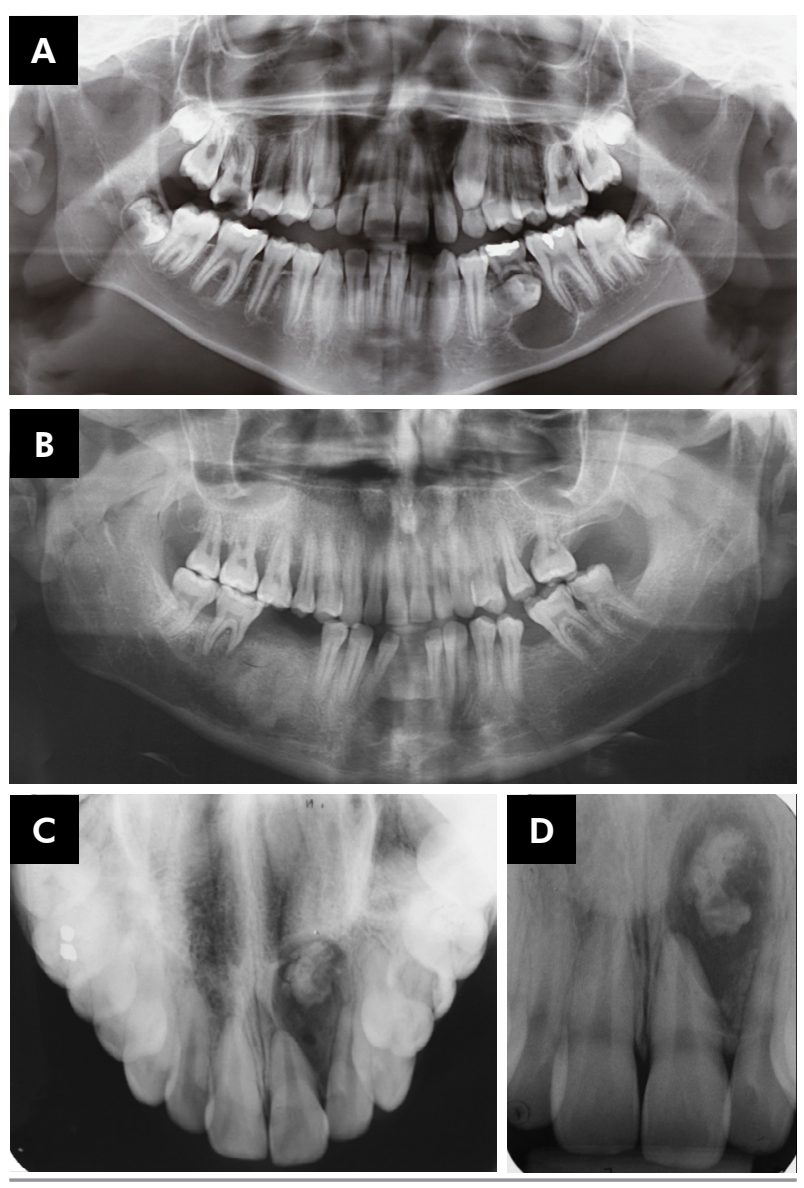

Figure 1. (A) Panoramic $x$-ray showing a radiolucent lesion involving the crown of the tooth 35 (Dentigerous cyst). (B) Poor delimited radiopaque lesion in right mandible (osteosarcoma). $(C, D)$ An occlusal and periapical x-ray showing a mist lesion involving the anterior region of the maxilla (calcifying cystic odontogenic tumor).
Consequently, there was no histopathological confirmation in the 47 lesions (32.86\%). Of these, 16 were osseous dysplasia, 9 were bone sclerosis, and 5 were condensing osteitis; bone exostosis, fibrous dysplasia, multiple myeloma, Stafne bone defect and sinus hypoplasia accounted for 1 case each (Figure 2).

Of the 93 cases with histopathological analysis, 38 were periapical cysts, 12 dentigerous cysts, 9 odontomas (7 compounds and 2 complexes), 8 KOT, 6 residual cysts, 5 ameloblastomas and other lesions. There were only 2 cases of malignant tumors, which were osteosarcoma and mucoepidermoid carcinoma (Figure 3).

Odontoma was the most frequent odontogenic tumor observed, and of the 9 represented cases, 7 were compound (4 in the mandible: 2 in the symphysis, and 2 in the body. 3 in the maxilla: 2 in the anterior region and one in the posterior) and 2 were complex (both in the maxilla: 1 in the anterior region and 1 in the posterior region). In regards to the radiographic aspect, 3 were radiopaque, 6 were mixed, and all 9 were unilocular. KOT was the
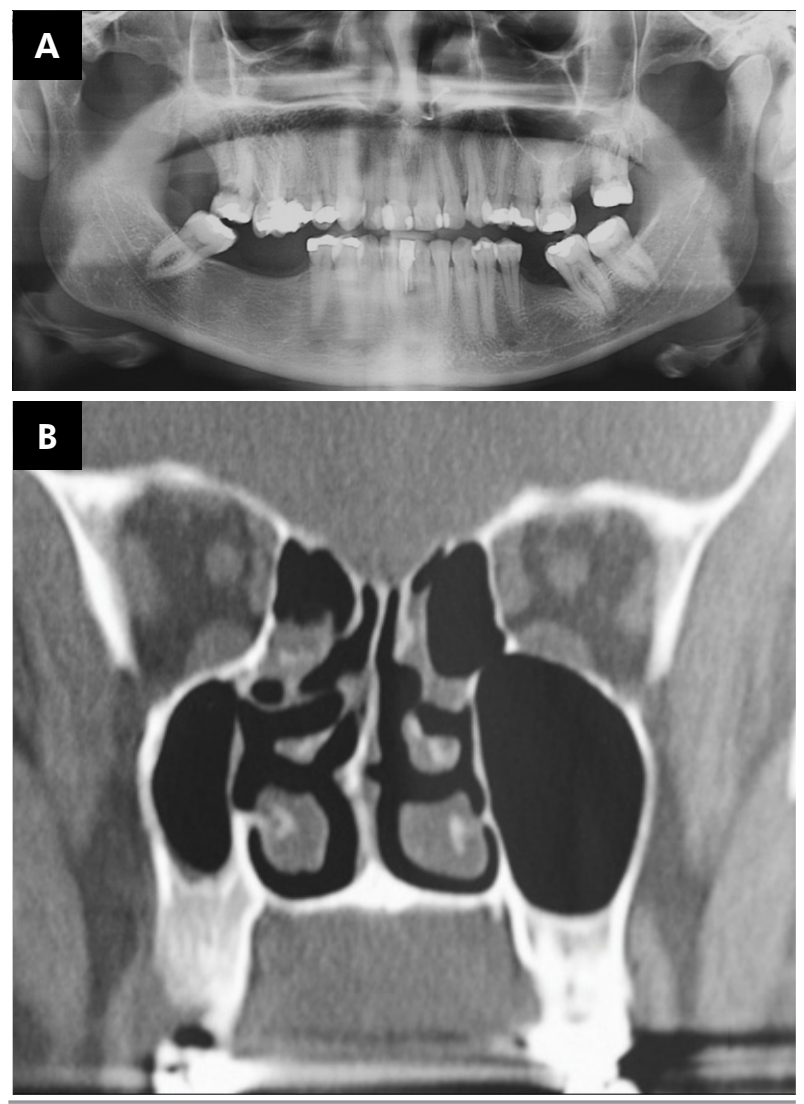

Figure 2. (A-C) Sinus hypoplasia observed in both panoramic $x$-ray and CT. The CT was crucial for the diagnosis. 


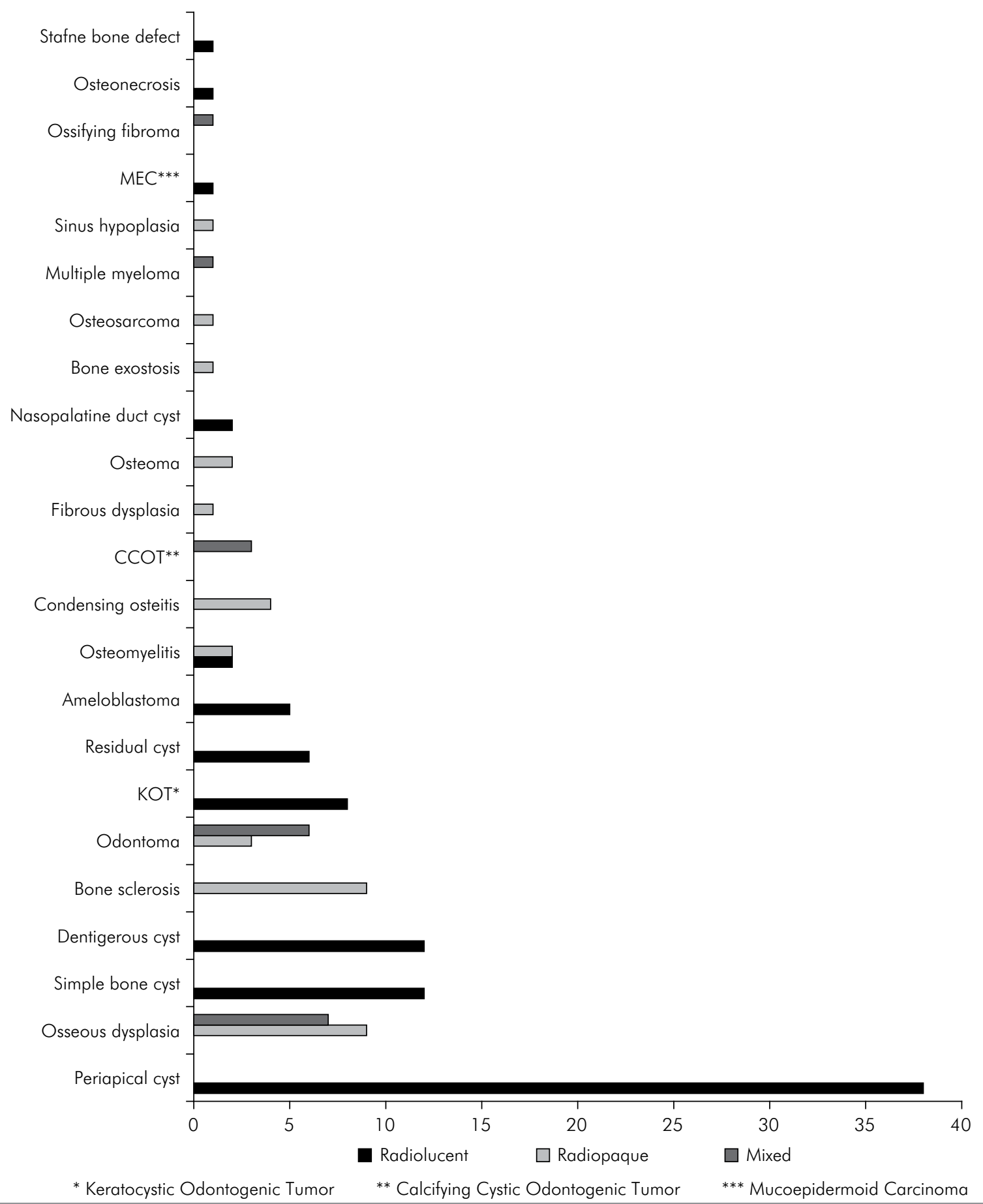

Figure 3. Correlation of final diagnosis of the 140 jaw lesions to radiographic aspect.

second most frequent odontogenic tumor observed and represented 8 cases; all of them were radiolucent and unilocular. The ameloblastoma was the third, which was observed in 5 cases. All of the cases were radiolucent, with 4 multilocular and 1 unilocular. Periapical cyst was the most frequent odontogenic cyst and represented 38 cases; all cases were radiolucent. Dentigerous cyst was the second most frequent with 12 cases. Malignant tumors represented 3 cases (osteosarcoma, mucoepidermoid carcinoma and multiple myeloma). All of the cases had swelling and cortical disruption, and the case of osteosarcoma presented paresthesia. 
Osseous dysplasia was observed in 16 cases, of which $11(68.75 \%)$ were classified as florid. Afro-descendent women were more affected, and the lesions had radiopaque and mixed aspect. Of the 16 lesions, 9 were exclusively radiopaque (5 florid, 3 focal and 1 periapical) and 7 were mixed ( 6 florid and 1 focal). A simple bone cyst was observed in 12 cases, and all of the cases affected the mandible. Bone sclerosis occurred in 9 cases. As well as simple bone cyst, all of the cases were radiographic findings.

In the Group 1, 79 out of 93 lesions (76.82\%) had the main clinical-radiographic diagnosis hypothesis confirmed. In Group 2, a total of 12 lesions had a surgical intervention, but there was no material to be collected. In addition, this characteristic associated to the clinical-radiographic feature was strongly indicative of the diagnosis of SBC. The diagnosis hypothesis was corrected in 11 of the cases (91.67\%) (Table 2). The rate of correct diagnosis including Group 1 and 2 was $85.7 \%$.

Table 2. Comparison between the diagnosis hypothesis to the final diagnosis.

\begin{tabular}{|c|c|c|c|}
\hline \multirow{2}{*}{ Final diagnosis } & \multicolumn{2}{|c|}{ Diagnosis hypothesis } & \multirow{2}{*}{ Total } \\
\hline & Correct (\%) & Incorrect (\%) & \\
\hline Periapical cyst & $37(97.37)$ & $1(2.63)$ & 38 \\
\hline Dentigerous cyst & $11(91.67)$ & $1(8,33)$ & 12 \\
\hline Simple bone cyst & $11(91.67)$ & 1 (8.33) & 12 \\
\hline Odontoma & $9(100)$ & $0(0)$ & 9 \\
\hline $\mathrm{KOT}^{\mathrm{a}}$ & $4(50)$ & $4(50)$ & 8 \\
\hline Residual cyst & $5(83.33)$ & $1(16.67)$ & 6 \\
\hline Ameloblastoma & $4(80)$ & $1(20)$ & 5 \\
\hline Osteomyelitis & $1(25)$ & $3(75)$ & 4 \\
\hline $\mathrm{CCOT}^{\mathrm{b}}$ & $1(33.33)$ & $2(66.66)$ & 3 \\
\hline Nasopalatine duct cyst & $2(100)$ & $0(0)$ & 2 \\
\hline Osteoma & $2(100)$ & $0(0)$ & 2 \\
\hline $\mathrm{MEC}^{\mathrm{c}}$ & $0(0)$ & $1(100)$ & 1 \\
\hline Ossifying fibroma & $1(100)$ & $0(0)$ & 1 \\
\hline Osteonecrosis & $1(100)$ & $0(0)$ & 1 \\
\hline Osteosarcoma & $1(100)$ & $0(0)$ & 1 \\
\hline
\end{tabular}

a: Keratocystic odontogenic tumor; ${ }^{\text {b: }}$ Calcifying cystic odontogenic tumor; c: Mucoepidermoid carcinoma.

\section{Discussion}

Most JL studies are retrospective, and they usually report data from histopathology labs. However, some entities (osseous dysplasias, bone sclerosis and others) do not require a biopsy to confirm the diagnosis. Consequently, these lesions may be underreported in the literature. In addition, JL represent a wide spectrum of diseases/alterations, and epidemiological studies simultaneously evaluating all of these lesions are uncommon. In general, the data are presented according to the following groups: odontogenic cysts and tumors ${ }^{3,8,9,10}$, fibro-osseous lesions ${ }^{11,12,13}$, malignant tumors ${ }^{14,15,16,17}$, and osteonecrosis ${ }^{18}$. The present study was prospectively designed with the purpose of determining the prevalence of these types of lesions. Additionally, the main radiographic features were assessed, and the data may be important to elaborate strategies for patient care and teaching in undergraduate programs.

A retrospective study revised data from 40,000 oral biopsies performed in the Canadian population during a period of 26 years. Cysts and odontogenic tumors consisted of 7,324 lesions (18.31\%). Interestingly, the authors emphasized that the small number of OD observed represented a bias due to the reluctance to biopsy this condition..$^{19}$ In the present study, 450 patients were observed for the first time during the 15 months of the study, and 130 of them $(29 \%)$ had JL. It is worth noting that there was no histopathological examination in 47 cases (44 patients), which represents approximately a third of all cases. Of these, the diagnosis was performed through clinical and radiographic features in 35 cases, and a surgical procedure was indicated, but there was no material to be collected in the remaining 12 cases. According to the clinical, radiographic, and surgical intervention features, these cases were diagnosed as simple bone cysts. The remaining 93 cases had their diagnosis confirmed by histopathological examination. Cysts (both non-odontogenic and odontogenic) and odontogenic tumors were the main lesions with $58(41.4 \%)$ and 25 cases $(17.8 \%)$, respectively. Periapical (38 cases $-65.5 \%$ ) and dentigerous cysts (12 cases $-20.7 \%$ ) were the most frequent. A retrospective study evaluated 418 cases of odontogenic cysts in the period from 1997 to 2006 
and found that 210 cases were periapical cysts (50.2\%) and $91(21.8 \%)$ were dentigerous cysts. ${ }^{9}$

There are a large number of odontogenic tumors; more than 20 types according to the WHO (2005). Some studies have shown ameloblastoma ${ }^{2,3,20}$ to be the most common tumor, whereas odontoma has been more frequent in others ${ }^{21,22}$. Interestingly, Fregnani et al. ${ }^{5}$ emphasized that odontoma is more common in colleges and ameloblastoma is more common in hospital. One possible explanation for this observation is that ameloblastoma treatment requires a more extensive surgery under general anesthesia. Thus, there is a difference in the order of odontogenic tumor prevalence when the studies are in colleges or hospitals. In this prospective study, 25 odontogenic tumors were diagnosed over a period of 15 months. There was a slight predominance of odontomas (9 cases). However, KOT and ameloblastoma also occurred frequently, in 8 and 5 cases, respectively.

It is well accepted that the mandible is the bone most commonly affected by JL (mainly in its posterior region). In a study conducted by Núñez-Urrutia et al. ${ }^{9}$, $61.5 \%$ of bone lesions occurred in the mandible. Paradental cysts $(97.7 \%)$ and dentigerous cysts (69.2\%) were observed more in the mandible, while radicular cysts were observed more in the maxilla (56.2\%). Similarly, our study showed that the mandible was more frequently affected (100 cases, $71.43 \%$ ), mainly the body region (71 cases). Dentigerous cysts (100\%) and residual cysts $(66.67 \%)$ were more common in the mandible, and radicular cysts (57.9\%) were more common in the maxilla.

A lab retrospective study at Thailand University evaluated 590 odontogenic tumors over a period of 34 years. The mandible was the main site for ameloblastoma, complex odontoma, KOT and calcifying cystic odontogenic tumors. However, the maxilla was more affected by compound odontomas and adenomatoid odontogenic tumors ${ }^{23}$. Similarly, Gupta et al..$^{20}$ observed that ameloblastoma, ameloblastic fibroma and complex odontoma mainly affect the mandible, and compound odontoma and adenomatoid odontogenic tumors mainly the maxilla. In our study, ameloblastoma (5 cases $-100 \%$ ), KOT (7 out of 8 cases $-87.5 \%$ ), compound odontoma (4 out of 7 cases $-57.14 \%$ ), and calcifying cystic odontogenic tumors (2 out of $3-66.66 \%$ ) more frequently occurred in the mandible. In contrast, the maxilla was more frequently affected by complex odontoma ( $100 \%$, only 2 cases).

An important radiographic feature of KOT is its anterior-posterior growth. Furthermore, many studies using both panoramic and occlusal x-rays data have not reported bone expansion. ${ }^{1}$ In contrast, studies using CT have described a slight expansion of both the buccal and lingual cortical bone. ${ }^{24,25}$ In the present study, there was $\mathrm{CT}$ information in 2 out of $8 \mathrm{KOTs}$, and both cases presented discrete expansion of the mandible. However, the basal cortical bone was not affected. Interestingly, in the other 2 cases (sinus hypoplasia and Stafne's bone defect), the CT was fundamental for the diagnosis and clinical management. For the former lesion, osseous dysplasia was the main diagnosis hypothesis in the panoramic x-ray, but after $\mathrm{CT}$, it was determined that the maxilla was normal and a reduction of the size of the maxillary sinus was observed (Figure 2). Consequently, biopsy was not indicated. However, in the other case, the Stafne's bone defect had been hypothesized in the x-ray, and the CT was essential to confirm the hypothesis.

Osseous dysplasia was observed in 16 patients (mean age of 50.4 years, range 28-77), of whom 12 were women (9 Afro-descendants) and 4 men (3 Afro-descendants). MacDonald-Jankowski ${ }^{26}$ previously conducted a systematic review of 158 cases of florid osseous dysplasia. The author found that 95\% of the cases occurred in Afro-descendants and that $97 \%$ in middle-aged women. In a Brazilian retrospective study, a total of 143 fibro-osseous lesions were diagnosed over a period of 9 years. The focal OD were predominantly radiopaque, whereas florid/periapical OD were predominantly mixed..$^{13}$ In our study, panoramic $x$-ray was used to diagnosis all of the cases of OD. In all of the cases, the diagnosis was only clinical and radiographic. Of the 11 cases of florid OD, 8 only affected the mandible while 3 affected both the mandible and the maxilla. The other OD cases affected only the mandible. Previous studies also showed that the mandible was more involved than the maxilla. ${ }^{11,26}$ In addition, most of the cases were discovered as incidental findings (i.e., there were no symptoms, and biopsy was unnecessary). 
Malignancies of the jaw are infrequent. Of the 140 cases diagnosed in this study, only 3 were malignant tumors (osteosarcoma, multiple myeloma and MEC). The osteosarcoma presented as a poorly defined radiopaque image, which caused enlargement of the periodontal ligament and root resorption (panoramic x-ray). Furthermore, the occlusal x-ray showed a "sunburst" pattern. The multiple myeloma was a multilocular radiolucent lesion with disruption of the cortical bone. The other case was a MEC of the maxillary sinus, and the lesion was radiolucent, unilocular, and caused root resorption.

In conclusion, JL are common and represented approximately $30 \%$ of all new cases diagnosed at CDO-FOUSP. The main diagnosed lesions were

\section{References}

1. Neyaz Z, Gadodia A, Gamanagatti S, Mukhopadhyay S. Radiographical approach to jaw lesions. Singapore Med J. 2008;49(2):165-76.

2. Arotiba JT, Ogunbiyi JO, Obiechina AE. Odontogenic tumours: a 15-year review from Ibadan, Nigeria. Br J Oral Maxillofac Surg. 1997;35(5):363-7. doi: 10.1016/S0266-4356(97)90411-3

3. Adebayo ET, Ajike SO, Adekeye EO. A review of odontogenic tumors in Kaduna, Nigeria. J Oral Maxillof Surg. 2005;63(6):811-9. doi:10.1016/j.joms.2004.03.022

4. Costa DOP, Maurício AS, Faria PAS, Silva LE, Mosqueda-Taylor A, Lourenço SQC. Odontogenic tumors: A retrospective study of four Brazilian diagnostic pathology center. Med Oral Patol Oral Cir Bucal. 2012;17(3):e389-94. doi:10.4317/medoral.17630

5. Fregnani ER, Fillipi RZ, Oliveira CR, Vargas PA, Almeida OP. Odontomas and ameloblastomas: variable prevalences around the world? Oral Oncol. 2002;38(8):807-8. doi:10.1016/S1368-8375(02)00050-7

6. Kramer IRH, Pindborg JJ, Shear M. Histological typing of odontogenic tumours. 2nd ed. Berlin: Springer; 1992.

7. Barnes L, Eveson JW, Reichart P, Sidransky D, editors. Pathology and genetics: head and neck tumours. Lyon: IARC Press; 2005. Chapter 6, Odontogenic tumours; p. 283-328. (World Health Organization classification of tumours).

8. Mosqueda-Taylor A. New findings and controversies in odontogenic tumors. Med Oral Patol Oral Cir Bucal. 2008;13(9):E555-8.

9. Nuñez-Urrutia S, Figueiredo R, Gay-Escoda C. Retrospective clinicopathological study of 418 odontogenic cysts. Med Oral Patol Oral Cir Bucal. 2010;15(5):e767-73. doi:10.4317/medoral.15.e767 periapical cyst, osseous dysplasia, dentigerous cyst and simple bone cyst. The most common radiographic features observed were radiolucent and unilocular. Biopsy was indicated for $66 \%$ of the cases. In the other cases, the diagnosis was concluded through clinical and radiographic features and surgical intervention (only for simple bone cysts).

\section{Acknowledgments}

The authors are grateful to the Brazilian Federal Agency for the Support and Evaluation of Graduate Education - CAPES and the National Council for Scientific and Technological Development - CNPq for their financial support. Dr. Fabio Alves is a CNPq research fellow.

10. Avelar RL, Primo BT, Pinheiro-Nogueira CB, Studart-Soares EC, Oliveira RB, Romulo de Medeiros J et al. Worldwide incidence of odontogenic tumors. J Craniofac Surg. 2011;22(6):2118-23. doi:10.1097/SCS.0b013e3182323cc7

11. Macdonald-Jankowski DS. Focal cemento-osseous dysplasia: a systematic review. Dentomaxillofac Radiol. 2008;37(6):350-60. doi:10.1259/dmfr/31641295

12. Worawongvasu R, Songkampol K. Fibro-osseous lesions of the jaws an analysis of 122 cases in Thailand. J Oral Pathol Med. 2010;39(9):703-8. doi:10.1111/j.1600-0714.2010.00932.x

13. Santos Netto JN, Cerri JM, Miranda AMM, Pires FR. Benign fibro-osseous lesions: clinicopathologic features from 143 cases diagnosed in an oral diagnosis setting. Oral Surg Oral Med Oral Pathol Oral Radiol. 2013;115(5):e56-65. doi:10.1016/j.oooo.2012.05.022

14. Perez DEC, Pires FR, Lopes MA, Almeida OP, Kowalski LP. Adenoid cystic carcinoma and mucoepidermoid carcinoma of the maxillary sinus: report of a 44-year experience of 25 cases from a single institution. J Oral Maxillofac Surg. 2006;64(11):1592-7. doi:10.1016/j.joms.2005.11.088

15. Bachar G, Goldstein D, Brown D, Tsang R, Lockwood G, Perez-Ordonez B et al. Solitary extramedullary plasmacytoma of the head and neck: long-term outcome analysis of 68 cases. Head Neck. 2008;3098):1012-9. doi:10.1002/hed.20821

16. Kamal M, Kaur P, Gupta R, Gupta S, Singh S. Mandibular plasmacytoma of jaw: a case report. J Clin Diagn Res. 2014;8(8):ZD20-1. doi:10.7860/JCDR/2014/9565.4674

17. Stewart BD, Reith JD, Knapik JA, Chi AC. Bone and cartilage-forming tumors and ewing sarcoma: an update with a gnathic emphasis. Head Neck Pathol. 2014;8(4):454-62. doi:10.1007/s12105-014-0587-8 
18. Marx RE. Osteoradionecrosis: a new concept of its pathophysiology. J Oral Maxillofac Surg. 1983;41(5):283-8. doi:10.1016/0278-2391(83)90294-X

19. Daley TD, Wysocki GP, Pringle GA. Relative incidence of odontogenic tumors and oral and jaw cysts in a Canadian population. Oral Surg Oral Med Oral Pathol. 1994;77(3):276-80. doi:10.1016/0030-4220(94)90299-2

20. Gupta B, Ponniah I. The pattern of odontogenic tumors in a government teaching hospital in the southern Indian state of Tamil Nadu. Oral Surg Oral Med Oral Pathol Oral Radiol Endod. 2010;110(11):e32-9. doi:10.1016/j.tripleo.2010.02.035

21. Ochsenius G, Ortega A, Godoy L, Peñafiel C, Escobar E. Odontogenic tumors in Chile: a study of 362 cases. J Oral Pathol Med. 2002;31(7):415-20. doi:10.1034/j.1600-0714.2002.00073.x

22. Buchner A, Merrell PW, Carpenter WM. Relative frequency of central odontogenic tumors: a study of 1,088 cases from Northern California and comparison to studies from other parts of the world. J Oral Maxillofac Surg. 2006;64(9):1343-52. doi:10.1016/j.joms.2006.05.019
23. Worawongvasu R, Tiensuwan M. Odontogenic tumors in Thailand: a study of 590 Thai patients. J Oral Maxillofac Surg Med Pathol. 2015;27(4):567-76. doi:10.1016/j.ajoms.2014.11.004

24. Apajalahti S, Hagström J, Lindqvist C, Suomalainen A. Computerized tomography findings and recurrence of keratocystic odontogenic tumor of the mandible and maxillofacial region in a series of 46 patients. Oral Surg Oral Med Oral Pathol Oral Radiol Endod. 2011;111(3):e29-37. doi:10.1016/j.tripleo.2010.10.010

25. Ariji $Y$, Morita M, Katsumata A, Sugita Y, Naitoh $\mathrm{M}$, Goto $\mathrm{M}$, et al. Imaging features contributing to the diagnosis of ameloblastomas and keratocystic odontogenic tumours: logistic regression analysis. Dentomaxillofac Radiol. 2011;40(3):133-40. doi:10.1259/dmfr/24726112

26. MacDonald-Jankowski DS. Florid cemento-osseous dysplasia: a systematic review. Dentomaxillofac Radiol. 2003;32(3):141-9. doi:10.1259/dmfr/32988764 